\title{
A CLASS OF EXPONENT-SUM TWO EQUATIONS OVER GROUPS
}

\author{
ANDREW CLIFFORD \\ The College of New Jersey, USA \\ e-mail: cliffan@tcnj.edu
}

(Received 20 June, 2000; accepted 2 February 2001)

\begin{abstract}
In this paper, relative pictures are used to analyze a certain family of exponent-sum two equations over groups.
\end{abstract}

2000 Mathematics Subject Classification. 20E05.

1. Introduction. If $G$ is a group, then an equation over $G$ is an element, $\epsilon=c_{1} t^{s_{1}} c_{2} t^{s_{2}} \ldots c_{k} t^{s_{k}}$, of the free product $G *\langle t\rangle$ in which the variable $t$ generates an infinite cyclic subgroup. The exponent sum of $\epsilon$ is $\sigma(\epsilon)=s_{1}+s_{2}+\ldots s_{k}$; the length of $\epsilon$ is $|\epsilon|=\left|s_{1}\right|+\left|s_{2}\right|+\ldots+\left|s_{k}\right|$. We say $\epsilon$ is solvable over $G$ if the inclusion induced homomorphism $G \rightarrow\langle G, t \mid \epsilon\rangle$ is injective. There are two main conjectures dealing with equations over groups. The Kervaire-Howie conjecture predicts that if $\sigma(\epsilon) \neq 0$ then $\epsilon$ is solvable over any group. In [10], Levin conjectured that any equation is solvable over a torsion free group. Interested readers are referred to [6], [7] and [8].

We will be interested in equations $\epsilon_{k}$ defined by

$$
\epsilon_{k}=a_{1} t^{-1} a_{2} t a_{3} t^{-1} a_{4} t \ldots a_{k-1} t^{-1} a_{k} t x t b_{1} t^{-1} b_{2} t \ldots b_{k-1} t^{-1} b_{k} t y t
$$

for the even integer $k$. Clearly, $\left|\epsilon_{k}\right|=2 k+2$ and $\sigma\left(\epsilon_{k}\right)=2$. In this paper, we prove two theorems.

Theorem 1. Assume that the equation $\epsilon_{k}$ is not solvable over the group $G$. Then there is at most one $j$ such that $a_{j} \neq b_{j}$.

THEOREM 2. Let $G$ be a torsion-free group, and assume that $\epsilon_{k}$ is not solvable over G. Then:

(1) there is exactly one $j$ such that $a_{j} \neq b_{j}$;

(2) there is some positive integer $p$ and an isomorphism from the subgroup of $G$ generated by $a_{j}$ and $b_{j}$ onto the group of integers taking $a_{j}$ to $p$ and $b_{j}$ to $p+1$;

(3) $x \neq y$.

As mentioned above, both the Kervaire-Howie Conjecture and the Levin Conjecture predict that $\epsilon_{k}$ is solvable over every group. Theorems 1 and 2 prove that in many situations it is. The outstanding cases described by these two theorems do not seem to be accessible by the methods used in this paper. The author would like to take this opportunity to express his gratitude to the referee for several useful suggestions on improving this paper, in particular, for a direct proof of Lemma 2. 
In [10], Levin proved that the equation $\epsilon_{0}=x t y t$ is solvable over any group $G$. So we will assume that $k$ is positive.

It is a consequence of work done in [4], that if $\epsilon_{k}$ is not solvable over the torsion free group $G$, then there is some positive word in either $\left\{a_{1}, b_{1}^{-1}\right\}$ or $\left\{a_{k}, b_{k}^{-1}\right\}$ that represents the identity in $G$. We see that Theorem 2 implies that for each $i$, there is a positive word in $\left\{a_{i}, b_{i}^{-1}\right\}$ that represents the identity in $G$. Therefore, Theorem 2 is a stronger result for $\epsilon_{k}$ than that given in [4].

In [8], Howie used relative diagrams to investigate equations over groups. Work following this scheme can be found in [5], [7], and [9]. In this note, we make use of the dual notion of relative diagrams, relative pictures. We give a brief description of this technique below. Readers interested in a more thorough treatment are referred to [2] and [3].

Given an equation $\epsilon=c_{1} t^{s_{1}} c_{2} t^{s_{2}} \ldots c_{k} t^{s_{k}}$, the pattern, $P_{\epsilon}$, is a directed star graph embedded in $S^{2}$ whose center is the vertex $v$ and whose corners are labelled by the coefficients $c_{1}, \ldots c_{k}$ of $\epsilon . P_{\epsilon}$ has an edge directed away from $v$ for each occurrence of $t$ in $\epsilon$ and an edge directed toward $v$ for each occurrence of $t^{-1}$ in $\epsilon$ so that if one circles $v$ counter-clockwise reading $t$ for each edge leaving $v, t^{-1}$ for each edge entering $v$, and the corresponding coefficient for each corner label, one will read the equation $\epsilon$ up to cyclic conjugacy. The inverse pattern $\bar{P}_{\epsilon}$ is obtained by reflecting $P_{\epsilon}$ in the sphere; changing the orientation on each edge and inverting the corner labels. We see that up to cyclic conjugation, $\bar{P}_{\epsilon}=P_{\epsilon^{-1}}$. As an example, Figure A shows the pattern $P_{\epsilon_{4}}$.

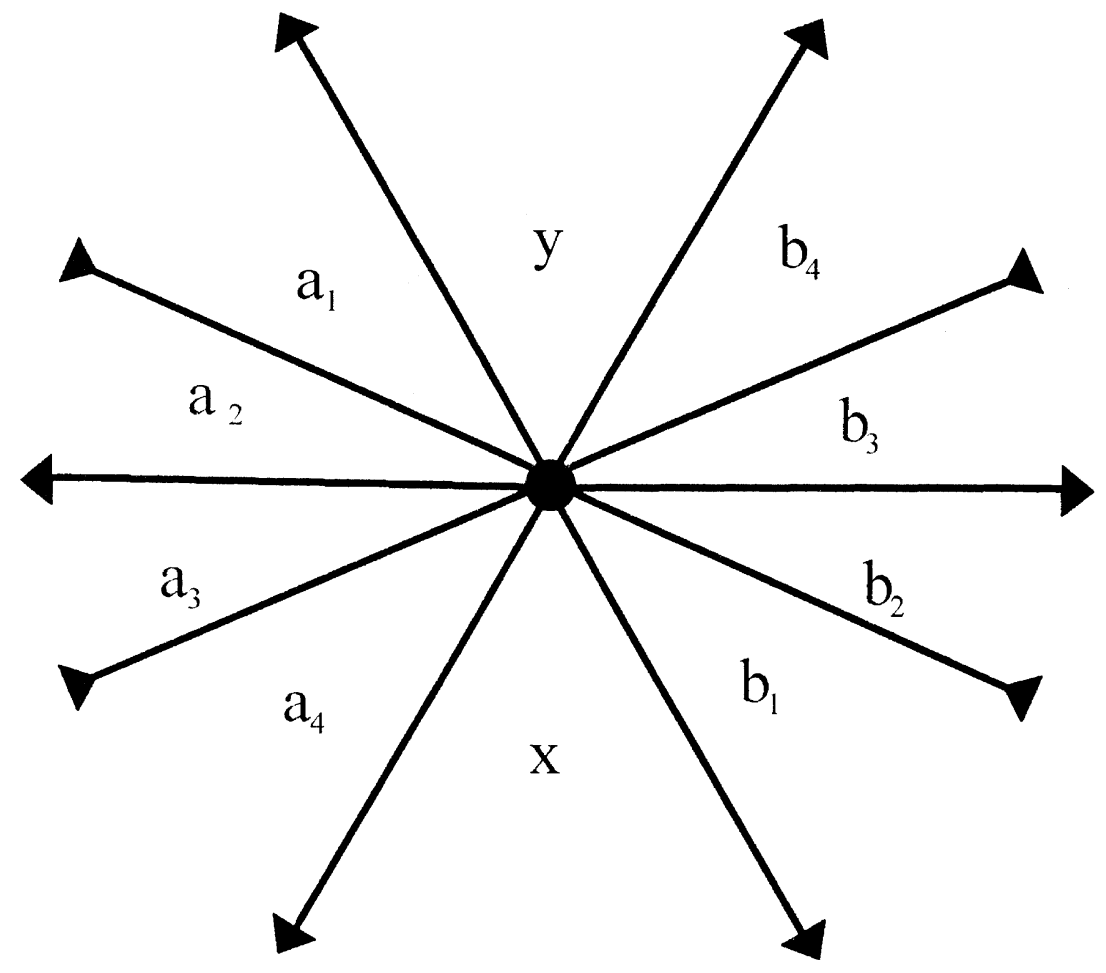

Figure A 
Given the equation $\epsilon$, a $P_{\epsilon}$-graph is a connected, directed graph embedded in $S^{2}$ whose corners are labelled so that each vertex $v$ looks either like $P_{\epsilon}$ (in which case $v$ is a positive vertex) or $\bar{P}_{\epsilon}$ (whence $v$ is negative). Figure B shows a $P_{\epsilon_{k}}$-graph with one positive vertex and one negative vertex.

Let $\epsilon$ be an equation and let $\Gamma$ be a $P_{\epsilon}$-graph. For each region $\Delta$ of $\Gamma$, define $\omega_{\Delta}$ as the word obtained by reading counter-clockwise around the corners of $\Delta$. Let $\omega_{\Delta}^{*}$ be the word obtained by reading clockwise around $\Delta$. These are well-defined up to cyclic conjugation. For example, if $\Gamma$ is the $P_{\epsilon_{k}}$-graph shown in Figure $\mathrm{B}$, and $\Delta$ is a region of $\Gamma$, then $\omega_{\Delta}=\left(a_{i} b_{i}^{-1}\right)^{ \pm 1}$ or $\left(x y^{-1}\right)^{ \pm 1}$ for some $i$. Clearly, if $\epsilon$ is an equation over the group $G$, then each $\omega_{\Delta}$ represents an element of $G$. We say that $\Gamma$ is reducible (in the sense of Sieradski [12]) if there is some region of $\Gamma$ whose corresponding word contains a cancelling pair, say $c_{i} c_{i}{ }^{-1}$. If $\Gamma$ is not reducible, then it is reduced.

The crux of our method is dual to that used by Howie in [8]. If we translate the work done by Howie into the dual, we get the following.

Theorem 3 (Howie). The equation $\epsilon$ is not solvable over the group $G$ if and only if there is a reduced $P_{\epsilon}$-graph with a specified region $\Delta_{0}$ such that:

(1) for each region $\Delta \neq \Delta_{0}$, the word $\omega_{\Delta}$ represents the identity of $G$;

(2) the word $\omega_{\Delta_{0}}^{*}$ does not represent the identity of $G$.

In this paper, we will assume that the equation $\epsilon_{k}$ is not solvable over some group $G$. Then we will see what types of regions must occur on reduced $P_{\epsilon_{k}}$-graphs. This will tell us what relations must hold in the group $G$.



Figure B 
Let $\epsilon$ be an equation and let $\Gamma$ be a $P_{\epsilon}$-graph. We say a region of $\Gamma$ is consistent if its boundary is consistently directed and each corner is labelled with the same coefficient up to exponent. In [3], the equation $\epsilon$ was defined to be Type $\mathrm{K}$ if each $P_{\epsilon}$-graph has at least two consistent regions. It follows from Howie's theorem that every Type K equation satisfies Levin's conjecture. In [9] and [3] it was shown that every exponent sum one equation is Type K. Figure B shows that, for any even $k$, the equation $\epsilon_{k}$ is not Type $\mathrm{K}$. In fact, these are the only exponent sum two equations that are known not to be type $\mathrm{K}$.

2. Proof of Theorem 1. For the remainder of this note, we will assume that the equation $\epsilon_{k}$ is not solvable over the group $G$ and that $\Gamma$ is a reduced $P_{\epsilon_{k}}$-graph whose existence is asserted by Howie's Theorem. We will let $\Delta_{0}$ be the specified region of $\Gamma$ so that if $\Delta \neq \Delta_{0}$, then $\omega_{\Delta}$ represents the identity of $G$. We also assume that, for all $i$, neither $a_{i}$ nor $b_{i}$ represent the identity in $G$. It follows that the only region which might be degree one is $\Delta_{0}$.

If $\kappa$ is a corner of some region of $\Gamma$ which is labelled with either $x$ or $y$, then $\kappa$ is a source corner; if $\kappa$ is labelled with either $x^{-1}$ or $y^{-1}$, then $\kappa$ is a sink corner. Following [3] and [4], we will add a family of dotted edges to $\Gamma$. Let $\Delta$ be any region of $\Gamma$ whose boundary is not consistently directed. We pair each source corner of $\Delta$ to a sink corner of $\Delta$. For each such pairing, we add a directed dotted edge from the source corner to the sink corner. We do this in such a way so that the added edges do not intersect. We call the resulting graph $\hat{\Gamma}$. We may now ignore the labels $x, y$, $x^{-1}$ and $y^{-1}$.

Let $\hat{\epsilon}_{k}$ be the string obtained from $\epsilon$ by replacing each of $x$ and $y$ with $s$. So, $\hat{\epsilon}_{k}=a_{1} t^{-1} a_{2} t \ldots a_{k} t s t b_{1} t^{-1} b_{2} t \ldots b_{k} t s t$. If we let dotted edges correspond to occurrences of $s$ in the same manner that solid edges correspond to occurrences of $t$, we see that $\hat{\Gamma}$ is a $P_{\hat{\epsilon}_{k}}$-graph where $P_{\hat{\epsilon}_{k}}$ is pictured in Figure C.

Now, the dotted edges of $\hat{\Gamma}$ make up a set of disjoint simple closed curves. These curves divide the ambient sphere into subsets which we call meta-regions. So, a metaregion is a surface embedded on the sphere whose boundary is a non-empty set of dotted curves and whose interior does not contain any dotted edges. There must be at least two meta-regions which are homeomorphic to disks. At least one of these does not properly contain the specified region $\Delta_{0}$. We call this meta-region $D$ and we call the dotted curve which is its boundary $C$.

We note that there are no vertices interior to $D$. Also, there are an even number of vertices on $C$. In fact, as one goes around $C$, one alternately reaches positive and negative vertices. We will restrict our attention to the circle graph $\Sigma=D \cap \hat{\Gamma}$. Any region of $\Sigma$ which is bounded by edges of $\Gamma$ reads a relation in $G$. Such a region will be called an interior region. No interior region has degree one, so $\Sigma$ has no loops.

A germ of an edge is a preferred small subinterval adjacent to one endpoint of that edge. So each edge has exactly two germs. Each vertex $v$ of $\Sigma$ is adjacent to $k+1$ solid germs and 2 dotted germs. If $v$ is a positive vertex, then $v$ either corresponds to the subword $\epsilon_{a}=s t a_{1} t^{-1} \ldots a_{k} t s$ of $\hat{\epsilon}$ or the subword $\epsilon_{b}=s t b_{1} t^{-1} \ldots b_{k} t s$. We say that $v$ is a positive $A$-vertex in the former case and a positive $B$-vertex in the latter. We weight the solid germs of $v$ with the set of integers $\{0,1, \ldots, k\}$ so that the germ counterclockwise (respectively, clockwise) of the corner labelled $a_{i}$ or $b_{i}$ is assigned the number $i-1$ (resp., $i$ ). We define negative $A$-vertices and negative $B$-vertices in the obvious way. We weight the germs at each negative vertex similarly 


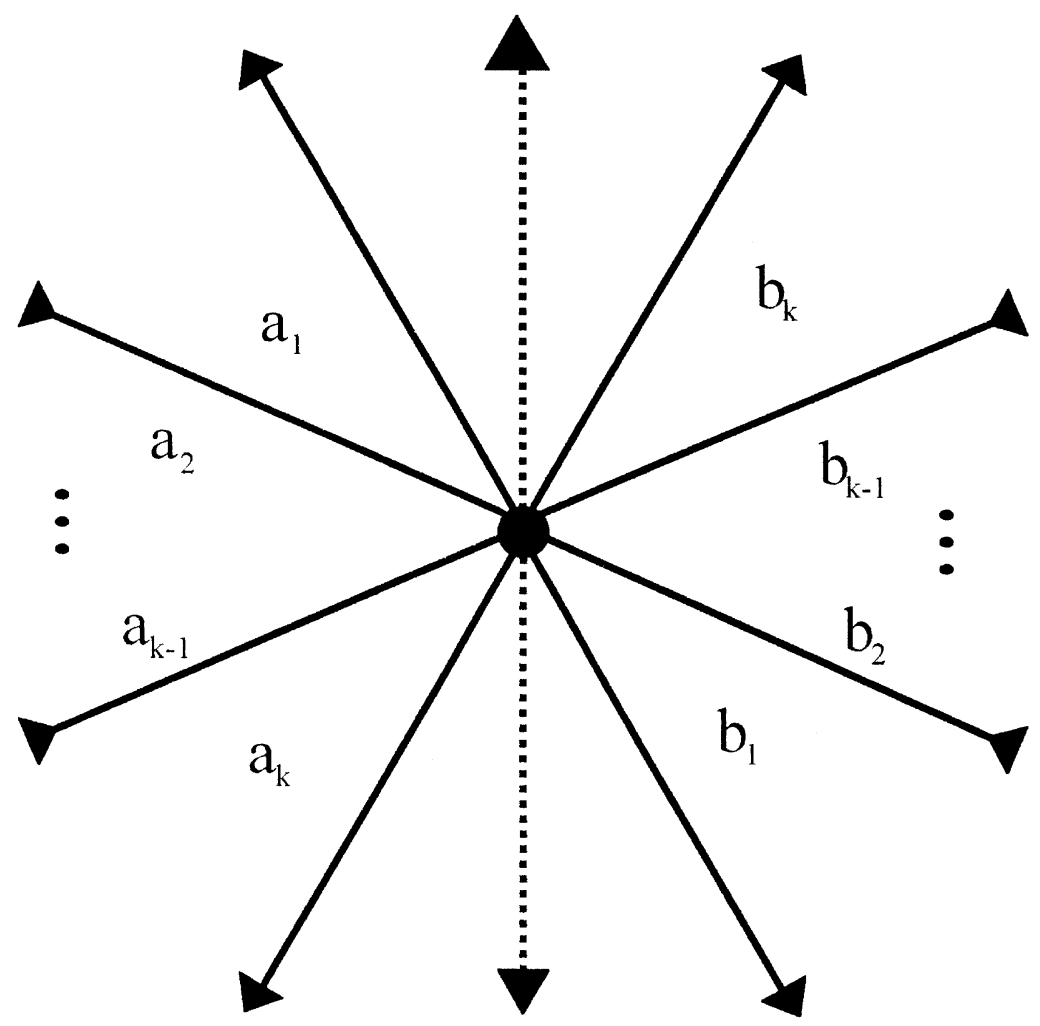

Figure C

using the set of non-positive integers $\{-k,-(k-1), \ldots, 0\}$. Let $e$ be an edge of $\Sigma$. The total weight, $\tau(e)$, of $e$ is the sum of the weights on its germs.

We measure the distance between two vertices of $\Sigma$ as the distance along $C$ when $C$ is given the graph metric. We define the spread of $v$ to be the maximum distance from $v$ to all vertices of $\Sigma$ to which $v$ is adjacent. It is not hard to see that there must be a vertex whose spread is 1 . Call this vertex $w$. Since $\Sigma$ has no loops, every edge adjacent to $w$ has weight 0 . Without loss of generality, we will assume that $w$ is a positive $A$-vertex.

Now, there are two cases: either there is a vertex $v$ whose distance from $w$ is 1 so that every edge adjacent to $w$ is also adjacent to $v$; or, there is some $j$ so that the edges whose germs at $w$ are weighted $\{0,1, \ldots, j-1\}$ are all adjacent to the vertex immediately clockwise of $w$ along $C$ and the edges whose germs at $w$ are weighted $\{j, \ldots, k\}$, are adjacent to the vertex immediately counter-clockwise of $w$.

In the first case, $w$ is adjacent to $k$ interior regions of degree two. These yield the relations $a_{i} b_{i}^{-1}$ for all $i$. In the second case, we obtain the relations $a_{i} b_{i}^{-1}$ for all $i \neq j$. In either case, there is at most one $j$ so that $a_{j} \neq b_{j}$. This ends the proof of Theorem 1.

\section{Proof of Theorem 2.}

Lemma 1. Let $\epsilon_{k}$ be an equation over the torsion free group $G$. If for all $i, a_{i}=b_{i}$, then $\epsilon_{k}$ is solvable over $G$. 
Proof. We factor the inclusion induced map $G \rightarrow\left\langle G, t \mid \epsilon_{k}\right\rangle$ as $G \rightarrow\langle G, s \mid x s y s\rangle \rightarrow$ $\left\langle G, s, t \mid x s y s, a_{1} t^{-1} a_{2} t \ldots a_{k} t s^{-1} t\right\rangle=\left\langle G, t \mid \epsilon_{k}\right\rangle$. That the first of these maps is injective is due to Levin [10]; that the second is is due to [3] and that each $a_{i}$ has infinite order.

The preceding lemma completes the proof of part 1 of Theorem 2. Let us fix the unique $j$ so that $a_{j} \neq b_{j}$. To see that the subgroup of $G$ generated by $a_{j}$ and $b_{j}$ is cyclic, we need to return to the techniques used in the previous section. So, let $D, C$ and $\Sigma$ be as above.

If every edge of $\Sigma$ has weight 0 , then for each $i$ there is an interior region $\Delta$ so that $\omega_{\Delta}=\left(a_{i} b_{i}^{-1}\right)^{p}$. (In fact, every interior region is of this form.) But, since these are relations in the torsion free group $G$, we have $a_{i}=b_{i}$ for all $i$, whence $\epsilon_{k}$ is solvable over $G$. So, there is at least one edge $e_{0}$ with $\tau\left(e_{0}\right) \neq 0$. We may assume that $e_{0}$ separates $D$ into two sets, one of which, say $D^{\prime}$, only contains edges which are weight 0 . Let $v$ and $w$ be the vertices adjacent to $e_{0}$.

We see that the sum of the weights of germs interior to $D^{\prime}$ is equal to 0 . It follows that $v$ and $w$ are either both positive or both negative. Furthermore, we see that the weights on the two germs of $e_{0}$ differ by 1 . Without loss of generality, we will assume that both $v$ and $w$ are positive and that $v$ is type A. Let $\Delta$ be the region in $D^{\prime}$ which has $e_{0}$ as an edge. Then $\Delta$ is an interior region and every edge of $\Delta$ other than $e_{0}$ has weight 0 . It follows that $\omega_{\Delta}=a_{i}\left(a_{i} b_{i}^{-1}\right)^{p}$ for some $i$ and $p$. Since this is a relation of $G$ we must have $i=j$. Moreover, $a_{j}=\left(b_{j} a_{j}^{-1}\right)^{p}$ and $b_{j}=\left(b_{j} a_{j}^{-1}\right)^{p+1}$. In particular, the subgroup of $G$ generated by $a_{j}$ and $b_{j}$ is cyclic generated by $b_{j} a_{j}^{-1}$. Part 2 of Theorem 2 follows.

Our last task is to see that $x \neq y$. This follows from the following two lemmas.

Lemma 2. Let $G$ be a torsion-free group with elements $c$ and $d$, not both of which are trivial. Let $\epsilon=c s d s$. Then $s$ has infinite order in the group $H$ presented $\langle G, s \mid \epsilon\rangle$.

Proof. Let $u=c s$. If $c=d, H$ has a free product decomposition as $H=G *\langle u\rangle$ where $\langle u\rangle$ is cyclic of order 2. If $c \neq d$, then $H$ has a decomposition as a free product with amalgamation as $H=G * \theta\langle u\rangle$ where $\langle u\rangle$ is infinite cyclic and $\theta$ is the isomorphism taking the subgroup of $G$ generated by $c^{-1} d$ onto the subgroup of $\langle u\rangle$ generated by $u^{2}$. In either case, the element $s=c^{-1} u$ is a cyclically reduced element of length 2 in this decomposition. It follows that $s$ has infinite order in $H$.

Lemma 3. Let $\epsilon_{k}$ be an equation over the torsion free group $G$. If $x=y$, then $\epsilon_{k}$ is solvable over $G$.

Proof. Assume that $x=y$. We only need to consider the case in which there is exactly one $j$ so that $a_{j} \neq b_{j}$. Without loss of generality, we assume that $j$ is even. Let $s=t a_{j+1} t^{-1} a_{j+2} t \ldots a_{k} t x t a_{1} t^{-1} a_{2} t \ldots t a_{j-1} t^{-1}$ and let

$$
\epsilon^{\prime}=a_{1} t^{-1} a_{2} t \ldots t a_{j-1} t^{-1} s^{-1} t a_{j+1} t^{-1} a_{j+2} t \ldots a_{k} t x t .
$$

With these substitutions, $\left\langle G, t \mid \epsilon_{k}\right\rangle=\left\langle G, s, t \mid a_{j} s b_{j} s, \epsilon^{\prime}\right\rangle$.

We factor the inclusion induced map $G \rightarrow\left\langle G, t \mid \epsilon_{k}\right\rangle$ as $G \rightarrow\left\langle G, s \mid a_{j} s b_{j} s\right\rangle \rightarrow$ $\left\langle G, s, t \mid a_{j} s b_{j} s, \epsilon^{\prime}\right\rangle$. Again, that the first map is injective is due to [10]; that the second is, is due to [3] and the fact that $s^{-1}$ has infinite order in $\left\langle G, s \mid a_{j} s b_{j} s\right\rangle$.

This ends the proof of Theorem 2 . 


\section{REFERENCES}

1. S. D. Brodskii and James Howie, One relator products of torsion-free groups, Glasgow Math. J. 35 (1993), 99-104.

2. W. A. Bogley and S. J. Pride, Aspherical relative presentations, Proc. Edinburgh Math. Soc. (2) 35 (1992), 1-39.

3. A. Clifford and R. Z. Goldstein, Tesselations of $S^{2}$ and equations over torsion-free groups, Proc. Edinburgh Math. Soc. (2) 38 (1995), 485-493.

4. A. Clifford and R. Z. Goldstein, Equations with torsion free coefficients, Proc. Edinburgh Math. Soc. (to appear).

5. M. Edjvet, Equations over groups and a theorem of Higman, Neumann, and Neumann, Proc. London Math. Soc. (3) 62 (1991), 563-589.

6. M. Edjvet and A. Juhasz, On equations over groups, Internat. J. Algebra and Comput. 4 (1994), 451-468.

7. S. M. Gersten, Reducible diagrams and equations over groups, in Essays in Group Theory (ed. S. M. Gersten), Math. Sci. Res. Inst. Publ. 8 (Springer, 1987), 15-73.

8. James Howie, The solution of length three equations over groups, Proc. Edinburgh Math. Soc. (2) 26 (1983), 89-96.

9. A. A. Klyachko, A funny property of sphere and equations over groups, Comm. Alg. 21 (1993), 2555-2575 604.

10. F. Levin, Solutions of equations over groups, Bull. Amer. Math. Soc. 68 (1962), 603-

11. W. Magnus, A. Karass and D. Solitar, Combinatorial Group Theory (Wiley Interscience Pub., New York, 1968).

12. A. J. Sieradski, A coloring test for asphericity, Quart. J. Math. Oxford (2) 34 (1983), 97-106.

13. J. R. Stallings, A graph-theoretic lemma and group embeddings, in: Combinatorial group theory and topology (ed. S. M. Gersten and J. R. Stallings) Ann. of Math. Stud. 111 (1987), 145-155. 